\title{
Parés pour la médecine intensive de demain
}

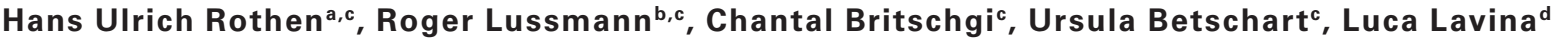 \\ ${ }^{a}$ Prof. Dr méd.; ${ }^{b}$ Dr méd.; ${ }^{c}$ Membres de la Société Suisse de Médecine Intensive (SSMI); ' Secrétariat général de la SSMI
}

Le congrès annuel de la Société Suisse de Médecine Intensive (SSMI) a réuni plus de 600 personnes à Interlaken, entre le 19 et le 21 septembre 2018. Comme les années précédentes, le congrès a débuté par un PreCongress-Day, une journée qui, avec son programme toujours très axé sur la pratique, est devenue partie intégrante du congrès annuel de la SSMI. C'est dans ce cadre qu'a eu lieu pour la quatrième fois le séminaire interdisciplinaire et interprofessionnel "ICU Management», un événement réservé aux personnels de direction des unités de soins intensifs suisses et à toutes les personnes intéressées par les aspects organisationnels du travail en médecine intensive. Cette année, l'événement était principalement consacré aux conditions-cadres financières et personnelles dans les unités de soins intensifs, un sujet important pour le personnel impliqué de près ou de loin dans cette spécialité.

\section{Des unités de soins intensifs sous-financées}

Dans une discipline telle que la médecine intensive impliquant un personnel nombreux et hautement qualifié et des infrastructures coûteuses pour le traitement de patient-e-s en état critique, les questions relatives au financement sont peut-être plus pertinentes que dans d'autres spécialités. Comme le constate Peter Rohner des HUG, la médecine intensive est généralement sous-financée en Suisse, et cette problématique est liée à un remboursement insuffisant des séjours très courts et plus longs que la moyenne dans les unités de soins intensifs. Selon Constanze Hergeth de SwissDRG, des rémunérations supplémentaires et le développement continu de ce système tarifaire permettront entre autres d'atténuer cette pression financière. Des rémunérations supplémentaires sont comptabilisées pour certaines prestations qui ne peuvent faire l'objet d'une facturation forfaitaire ou être assignées à un groupe de cas DRG. Toutefois, leur effet sur le financement n'est pas assez important pour couvrir les déficits et résoudre entièrement le problème du sous-financement. Outre cette différenciation et ce développement, Guido Schüpfer de l'hôpital cantonal de Lucerne considère les "taux de base» comme décisifs. SwissDRG définit si ceux-ci restent différenciés. Le montant du taux de base, c'est-à-dire le montant du remboursement par unité de pondération des coûts, est alors négocié entre les fournisseurs de services et les financiers.

\section{Les unités de soins intermédiaires améliorent les finances des unités de soins intensifs}

Selon Olga Endrich de l'hôpital de l'île à Berne, les unités de soins intermédiaires ou IMC participent aussi à l'amélioration perceptible du sous-financement de la médecine intensive. Ces unités se situent pour ainsi dire à l'interface entre les stations de lits traditionnelles et les unités de soins intensifs. Ce genre d'unité s'occupe typiquement de patient-e-s dont les fonctions vitales font l'objet d'une surveillance rapprochée, ou qui requièrent une prise en charge très lourde de la part des infirmiers, mais chez qui les fonctions vitales ne doivent être soutenues que dans une moindre mesure. En collaboration avec huit autres sociétés, la SSMI a pris l'initiative d'établir des normes pour ces IMC, de même qu'une procédure visant leur reconnaissance. Aujourd'hui, près de 40 IMC ont déjà été reconnues.

\section{Médecine intensive du futur?}

Compte tenu d'une pression des coûts toujours plus forte dans le système de santé, la charge qui pèse sur le personnel a tendance à augmenter. Des innovations techniques peuvent toutefois contribuer à soulager le personnel médical et infirmier de médecine intensive. Alexander König, fondateur d'une start-up allemande qui allie robotique et technique médicale, évoque un dispositif thérapeutique robotisé qui offre un soutien sûr aux patient-e-s lors de la mobilisation précoce. Contrairement à d'autres méthodes de mobilisation, ce système requiert moins de personnel et s'adapte aux besoins individuels des patient-e-s. Michael Czaplik de l'Uniklinik RWTH d'Aix-la-Chapelle évoque un projet pilote de télémédecine. Selon lui, la télémédecine peut 
également s'appliquer à la médecine intensive. Des méthodes de communication modernes pourraient contribuer à assurer la qualité des traitements dans l'unité de soins, tout en réduisant la dotation en personnel.

\section{Prendre des décisions basées sur les «Big Data»}

De la même manière, l'intelligence artificielle pourrait contribuer à soulager le personnel médical au quotidien, comme l'indique Patrick Schwab de l'ETH Zurich. Grâce à l'introduction de systèmes techniques qui analysent de gros volumes de données patient, personnel soignant et médecins sont en mesure de fonder leurs décisions sur ces données, ce qui permet d'économiser des ressources. S'ajoute à cela le fait que ces systèmes à capacité d'apprentissage gagnent en précision et en fiabilité à mesure qu'on les utilise. Malgré ces innovations qui facilitent le travail du personnel soignant et des médecins, les référents s'accordent à dire qu'à l'avenir, l'humain ne pourra pas être entièrement remplacé par la technique.

\section{Esprit d'équipe et responsabilité personnelle}

Si le travail aux soins intensifs, diversifié, est attrayant pour les médecins et pour le personnel soignant, les horaires de travail particuliers et les éprouvantes destinées auxquelles le personnel est souvent confronté rendent également cette spécialité très exigeante, comme le soulignent Sara Cereghetti des HUG et Philipp Eckert du CHUV. Le surmenage du personnel a des répercussions négatives sur la qualité des traitements, raison pour laquelle le personnel médical doit également veiller, pour lui-même, à apporter des soins de qualité aux patient-e-s. Dans ce contexte, il existe de nombreuses façons de lutter contre le surmenage. Comme l'indique Barbara Ricu des HUG, l'amélioration du travail d'équipe peut déjà contribuer à éviter les burnouts: une meilleure entraide mutuelle au sein du service et des décisions collégiales permettent d'échapper au surmenage. Comme le souligne Bjarte Rogdo de la clinique pédiatrique de Suisse orientale, une collaboration interprofessionnelle étroite telle qu'elle existe dans les unités de soins intensifs suisses peut améliorer le travail d'équipe. D'autre part, il est important aussi que l'ensemble des membres de l'équipe se sentent responsables et participent activement à la prise de décisions. Il est capital de trouver un équilibre entre soutien mutuel et responsabilité individuelle. Les cadres doivent également, comme l'affirme Barbara Ricou, créer des dispositifs susceptibles d'apporter une aide psychologique aux collaboratrices et collaborateurs dans des situations exceptionnelles tout comme au quotidien.

Muriel Joris-Frasseren de l'hôpital de Sion précise que dans des cas extrêmes, un surmenage excessif peut entraîner une pénurie de personnel contre laquelle on ne peut évidemment lutter durablement en recrutant du personnel à l'étranger. Pour les diverses institutions, des licenciements sont toujours synonymes de pertes financières, car l'engagement de personnel qualifié et la mise en place de formations continues coûtent cher. C'est aussi pour cette raison qu'il doit exister un intérêt pour la rétention des collaborateurs sur le long terme, comme le souligne Marie-Noëlle von Allmen Widmer, présidente de la Commission pour la formation post-graduée et continue des infirmières et infirmiers de la SSMI.

\section{Flexibilité des employeurs et employés}

Pour Manuel Gwiss de l'hôpital de Haute-Engadine, la flexibilisation des horaires de travail rigides déjà évoqués est l'une des solutions à envisager, car le personnel hospitalier aussi est de plus en plus demandeur d'un aménagement libre du temps de travail. Gery Brüderlin, ancien responsable du personnel d'UBS et enseignant à la FHNW, confirme cette observation et souligne son importance à une époque marquée par le changement démographique: comme la population suisse vieillit, il est presque inévitable de relever l'âge de la retraite. Il est donc de plus en plus important que les collaboratrices et collaborateurs continuent de se former, jusqu'à un âge avancé. Par ailleurs, les employeurs doivent imaginer de nouveaux modèles de travail flexibles, car bon nombre d'employés plus âgés ne peuvent plus ou ne souhaitent plus travailler de la même manière qu'au début de leur carrière. Ainsi, employeurs et employés devront faire preuve de plus de flexibilité à l'avenir, se montrer imaginatifs et innovants pour répondre aux défis de demain. 